\title{
Short-term regulation of the mammalian pyruvate dehydrogenase complex
}

\author{
Sławomir Strumiło \\ Department of Animal Biochemistry, Institute of Biology, University of Biatystok, Białystok, Poland
}

Received: 12 January, 2005; revised: 21 March, 2005; accepted: 19 April, 2005

available on-line: 11 July, 2005

\begin{abstract}
In this minireview the main mechanism of control of mammalian pyruvate dehydrogenase complex (PDHC) activity by phosphorylation-dephosphorylation is presented in the first place. The information recently obtained in several laboratories includes new data about isoforms of the PDH converting enzymes (kinase and phosphatase) and their action in view of short-term regulation of PDHC. Moreover, interesting influence of exogenous thiamine diphosphate (TDP) and some divalent cations, especially $\mathrm{Mn}^{2+}$, on the kinetic parameters of PDHC saturated with endogenous tightly bound TDP, is discussed. This influence causes a shortening of the lag-phase of the catalyzed reaction and a strong decrease of the $K_{\mathrm{m}}$ value of PDHC mainly for pyruvate. There are weighty arguments that the effects have an allosteric nature. Thus, besides reversible phosphorylation, also direct manifold increase of mammalian PDHC affinity for the substrate by cofactors seems an important aspect of its regulation.
\end{abstract}

Keywords: pyruvate dehydrogenase complex, regulation, phosphorylation, dephosphorylation, allosteric effect, thiamine diphosphate

The multienzyme pyruvate dehydrogenase complex (PDHC) catalyzes the conversion of pyruvate, $\mathrm{CoA}$ and $\mathrm{NAD}^{+}$into acetyl-CoA, NADH and $\mathrm{CO}_{2}$ (Reed, 2001). The main product, a CoA-activated two-carbon unit, can be condensed with oxalacetate in the first reaction of the citric acid cycle, or used for fatty acid and cholesterol synthesis. So, the several-step process of oxidative decarboxylation of pyruvate occupies a central position in cellular metabolism, because it links glycolysis with the citric acid cycle and lipid biosynthesis. In eukaryotic cells, the PDHC is located in the mitochondria matrix and may be regulated in numerous ways (Wałajtys et al., 1974; Wieland, 1983).

\section{PDHC STRUCTURE}

The mammalian PDHC is of great complexity. Its structural core is composed of 60 lipoatecontaining subunits of dihydrolipoamide acetyltransferase (EC 2.3.2.12; abbreviated E2) (Yeman, 1989; Reed, 2001). Each E2 subunit consists of an inner domain (ID), a binding domain (BD), and two lipoyl domains $\left(\mathrm{LD}_{1}, \mathrm{LD}_{2}\right)$ connected by flexible linkers (Fig. 1). The inner domains are arranged in a pentagonal dodecahedron and the others form an extended "swinging arm" including the lipoate residues (Perham, 2000). Thirty heterotetrameric $\left(\alpha_{2} \beta_{2}\right)$ molecules of TDP-containing pyruvate dehydrogenase (EC 1.2.4.1; abbreviated E1) are joined to the binding domains of E2 (Reed, 2001; Fries et al., 2003). Twelve homodimeric molecules of FAD-containing dihydrolipoamide dehydrogenase (EC 1.8.1.4; abbreviated E3) are connected with the binding domains of the so called "E3-binding protein" which differs from typical E2 subunit because it contains only one lipoyl domain $\left(\mathrm{LD}_{3}\right)$ and is not catalytically active (Harris et al., 2002; Hiromasa et al., 2004). Detailed information about the PDHC structure can be found in recently published reviews (Perham, 2000; Reed, 2001; Patel \& Korotchkina, 2003).

Studies of the structural organization of E1 may be especially useful for understanding its catalytic and regulatory mechanisms. For example, the crystal structure of the holo-form of human pyruvate dehydrogenase at $1.95 \AA$ resolution, recently determined by Ciszak et al. (2003), gives evidence

Abbreviations: BD, binding domain; E1, TDP-containing pyruvate dehydrogenase; E2, dihydrolipoamide acetyltransferase; E3, FAD-containing dihydrolipoamide dehydrogenase; ID, inner domain; $\mathrm{LD}_{1}$, $\mathrm{LD}_{2}$ and $\mathrm{LD}_{3^{\prime}}$ lipoyl domains; PDHC, pyruvate dehydrogenase complex; PDHK, pyruvate dehydrogenase kinase; PDHP, pyruvate dehydrogenase phosphatase; TDP, thiamine diphosphate. 
that a shuttle-like motion of E1 heterodimers $(\alpha \beta)$ is possible. This is in accordance with a flip-flop mechanism of the catalytic action of animal E1 (Khailova et al., 1990). Site-directed mutagenesis, kinetic analysis and X-ray crystallography of human E1 revealed that Pro-188, Met-181 and Arg-349 in the $\alpha$-subunit, and Trp-135 in the $\beta$-subunit play important structural roles. Moreover, the $\alpha$ Pro- 188 and $\alpha$ Met- 181 residues were found to be essential for binding of TDP (Korotchkina et al., 2004).

\section{REGULATION BY REVERSIBLE PHOSPHORYLATION}

Phosphorylation-dephosphorylation is undoubtedly the main mechanism of control of mammalian PDHC activity. It was discovered over 30 years ago (Linn et al., 1969; Wieland \& Jagow-Westermann, 1969) and has been intensively investigated ever since (Wałajtys et al., 1974; Strumiło et al., 1981; Wieland, 1983; Denton et al., 1996; Kolobova et al., 2001; Holness \& Sugden, 2003; Roche et al., 2003; Bao et al., 2004a). For realization of the mechanism, PDHC uses two specific enzymes: pyruvate dehydrogenase kinase (EC 2.7.1.99; PDHK) and pyruvate dehydrogenase phosphatase (no EC number; PDHP) Phosphorylation and concomitant inactivation of E1 is catalyzed by PDHK, whereas dephosphorylation and reactivation of E1 by PDHP (Linn et al., 1969; Wieland, 1983). The regulatory enzymes are both integrated with PDHC; they bind to PDHC mainly via the E2-lipoyl domains (Harris et al., 2002) (Fig. 1).

\section{CHARACTERIZATION OF PDH KINASES}

PDHK is a specific kinase that phosphorylates three serine residues in three separate sites of the E1-component of the PDHC, using ATP as a do-

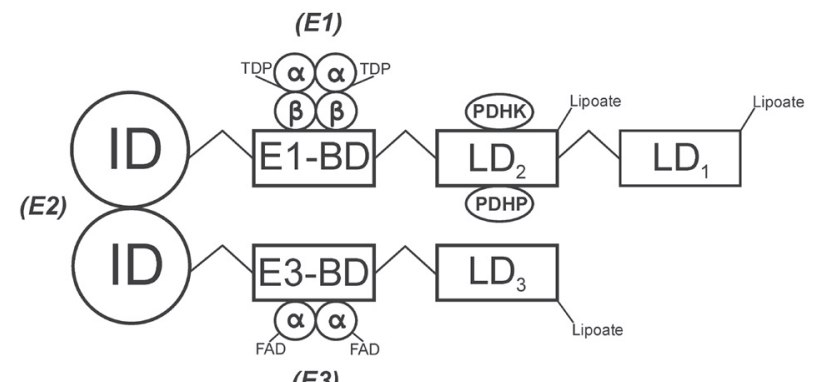

Figure 1. Scheme of a fragment of the mammalian pyruvate dehydrogenase complex structure (from Harris et al., 2002, modified).

Only two subunits of E2 with E1 and E3 components are shown. In complete PDHC 60 inner domains are arranged in a pentagonal dodecahedron. Abbreviations: ID, inner domain; BD, binding domain; LD, lipoyl domain. nor of phosphate group (Wieland, 1983; Reed, 2001; Kolobova et al., 2001; Bao et al., 2004a). Mammalian PDHK has been shown to exist in four isoforms (Gudi et al., 1995; Bowker-Kinley et al., 1998; Harris et al., 2002). It should be noted that genes which encode $\mathrm{PDHK}_{1}, \mathrm{PDHK}_{2}$ and $\mathrm{PDHK}_{4}$ are more strongly expressed in the muscle tissues and liver. On the other hand, genes encoding $\mathrm{PDHK}_{3}$ and $\mathrm{PDHK}_{2}$ are expressed in the kidney, brain, and testis (Harris \& Huang, 2001; Patel \& Korotchkina, 2003). This determines the respective differences in tissue distribution of mammalian PDHK isoforms and their mRNAs. Recently the three-dimensional structure of rat PDHK (isoform 2) has been established (Steussy et al., 2001). Interestingly, the mammalian PDHKs exhibit low homology with other eukaryotic serine protein kinases, but are similar to bacterial histidine protein kinases (Harris et al., 2002). However, their catalytic mechanisms are different, because PDHKs phosphorylate a serine residue directly, without phosphorylation of a histidine intermediate (Steussy et al., 2001). Each PDHK isoform is a homodimer composed of subunits of molecular masses between 39 and 48 kDa (Patel \& Korotchkina, 2003).

\section{REGULATORY PROPERTIES OF PDH KINASES}

Mammalian PDHC contains only 1-3 molecules of PDHK (Yeaman, 1989) which bind mainly to the mobile lipoyl domains of E2-subunits (Liu et al., 1995). Only the $\mathrm{PDHK}_{4}$ isoform prefers the lipoyl domain of the "E3-binding protein" (Roche et al., 2003). Such localization of PDHKs on the "swinging arms" and their transfer from one lipoyl domain to another gives the possibility of reaching almost all phosphorylation sites in the 30 molecules of E1 (Korotchkina \& Patel, 2001). PDHKs are activated via conformational changes when bound to the lipoyl domains (Roche et al., 2003; Bao et al., 2004b). Moreover, the activity of PDHK is stimulated by the reduction and acetylation of the lipoyl residues occurring during PDC action (Harris et al., 2002). Numerous studies with PDHC isolated from various mammalian tissues indicate that PDHK is stimulated by NADH and acetyl-CoA (Wieland, 1983; Yeaman, 1989; Reed, 2001). A high $\mathrm{NADH} / \mathrm{NAD}^{+}$ratio causes reduction of the lipoyl groups through the reversal of the reaction catalyzed by dihydrolipoamide dehydrogenase (E3), and a high acetyl-CoA/CoA ratio promotes acetylation of the reduced lipoyl groups by E2 (Patel \& Korotchkina, 2003). High NADH and acetylCoA concentrations are found in mitochondria when fatty acid oxidation is intensive, for example during starvation, in high fat diet and diabetes ( $\mathrm{Wu}$ et al., 2000; Harris et al., 2002). Phosphorylation and concomitant inactivation of PDHC by stimulated PDHK is very typical for such metabolic states. On the oth- 
er hand, high $\mathrm{NAD}^{+}$and $\mathrm{CoA}$ concentrations lead to PDHK inhibition, and the PDHC remains active (Wieland, 1983; Holness \& Sugden, 2003). Pyruvate also exerts an inhibitory influence on PDHK (Bao et al. 2004a). Isoform $\mathrm{PDHK}_{2}$ is more sensitive to inhibition by pyruvate and stimulation by NADH and acetyl-CoA than the other ones (Harris et al., 2002; Bao et al., 2004a). Competitive inhibition of PDHK by ADP versus ATP was also considered to be essential in the regulation of the PDHC (Strumiło et al., 1981; Wieland, 1983). Studies of $\mathrm{PDHK}_{2}$ showed that ADP dissociation from active sites is a limiting step in E2-activated catalysis by isoform 2 (Bao et al., $2004 b)$. Conversion of all lipoyl groups in the E2oligomer to the oxidized form greatly reduced $k_{\text {cat }}$ and the $K_{\mathrm{m}}$ of $\mathrm{PDHK}_{2}$ for ATP. On the other hand, reduction and, to a greater extent, reductive acetylation of the lipoyl groups increased $\mathrm{PDHK}_{2}$ binding to E2 and stimulated it (Bao et al., 2004a). Thus, fluctuations of pyruvate concentration and the NADH/ $\mathrm{NAD}^{+}$, acetyl-CoA/CoA and ATP/ADP ratios in varied hormonal and nutritional states significantly change PDHK activity and, in an opposite manner, the activity of the PDHC. It should also be added that a lipoprotein which strongly inhibits the PDHC has been isolated from bovine adrenal mitochondria (Strumiło et al., 1984). The degree of inhibition was higher at lower ATP concentrations. It was proposed that the mitochondrial lipoprotein could specifically shield the active site of PDHK or the phosphorylation sites of E1 during catalytic action.

As presented above, the E1-component of mammalian PDHC has three phosphorylation sites. Isoforms of PDHK show different activity during phosphorylation of the sites. Their relative activities toward site 1 are: $\mathrm{PDHK}_{2}>\mathrm{PDHK}_{4}>\mathrm{PDHK}_{1}>$ $\mathrm{PDHK}_{3}$; toward site 2: $\mathrm{PDHK}_{3}>\mathrm{PDHK}_{4}>\mathrm{PDHK}_{2}>$ PDHK $_{1}$ (Patel \& Korotchkina, 2003). In the case of site 3, the differences in the activity of the PDHK isoforms are not so clear. According to Kolobova et al. (2001), $\mathrm{PDHK}_{1}$ can phosphorylate all three sites, whereas $\mathrm{PDHK}_{2}, \mathrm{PDHK}_{3}$ and $\mathrm{PDHK}_{4}$ each phosphorylates only sites 1 and 3 . Earlier, it was reported that only phosphorylation at sites 1 and 2 results in PDHC inactivation, yet additional phosphorylation of site 3 inhibits reactivation of E1 by $\mathrm{PDH}$ phosphatase (Wieland, 1983). However, according to new data, phosphorylation of either site leads to PDHC inactivation, but in a different way (Korotchkina \& Patel, 2001; Kolobova et al., 2001). For example, phosphorylation of site 1 of human pyruvate dehydrogenase prevents its interaction with the substrate, pyruvate, and a lipoyl domain, whereas modification of site 3 hinders E1 interaction with TDP (Patel \& Korotchkina, 2003). Conversely, exogenous TDP added to the reaction medium results in inhibition of PDHK activity (Walsh et al., 1976; Czygier \& Strumiło, 1995a). TDP especially decreases the amount of phosphate that $\mathrm{PDHK}_{1}$ incorporates in sites 2 and 3 , and $\mathrm{PDHK}_{2}$ in site 2 (Kolobova et al., 2001). Interesting data were obtained in kinetic investigation of aurochs heart PDHC: completely dephoshporylated PDHC showed a positive cooperativity of pyruvate binding sites at low substrate concentrations, yet partial phosphorylation of the complex (to $35 \%$ reduction of its activity) abolished the cooperativity (Czygier \& Strumiło, 1995b).

Besides the short-term regulation by the metabolites, a long-term control of PDHK amount at the transcriptional level is very important. This aspect is presented thoroughly in the recently published reviews (Harris et al., 2002; Patel \& Korotchkina, 2003). In this minireview, only several examples of long-term regulation will be quoted. Of the four PDHK isoforms greater changes in the amount of enzyme protein were observed for $\mathrm{PDHK}_{4}$ and $\mathrm{PDHK}_{2}$. Starvation and diabetes increase the level of $\mathrm{PDHK}_{4}$ expression in most tissues (Harris et al., 2001). Insulin treatment of diabetic rats reverses the changes (Harris et al., 2002). Starvation also increases $\mathrm{PDHK}_{2}$ expression in the liver and kidney (Wu et al., 2000).

\section{CHARACTERIZATION OF PDH PHOSPHATASES}

PDHP is a specific phosphatase that removes $\mathrm{P}_{\mathrm{i}}$ from phosphorylated sites of $\mathrm{E} 1$ and reactivates the component and the whole PDHC. Mammalian PDHP consists of two nonidentical subunits of 52 and $96 \mathrm{kDa}$ (Chen et al., 1996). The smaller subunit performs the catalytic function, whereas the larger one is a flavoprotein performing a regulatory function (Lawson et al., 1997). There are two isoforms of PDHP encoded by different genes for the catalytic subunits (Huang et al., 2003). The isoforms PDHP and $\mathrm{PDHP}_{2}$ show some differences in activity, regulation, and tissue distribution (Karpova et al., 2003). It is known that binding of $\mathrm{PDHP}_{1}$ to the mammalian PDHC occurs thorough the lipoyl domain $\mathrm{LD}_{2}$ and requires the presence of $\mathrm{Ca}^{2+}$ which plays a bridging role (Roche et al., 2003). As with the binding of $\mathrm{PDHP}_{2}$, the existing information is insufficient for unequivocal conclusions.

\section{REGULATORY PROPERTIES OF PDHP}

The reaction catalyzed by both isoforms of PDHP requires $\mathrm{Mg}^{2+}$ concentrations in the millimolar range (Linn et al., 1969; Reed, 2001). Magnesium ions may be replaced by $\mathrm{Mn}^{2+}$. The $K_{\mathrm{m}}$ values of pig heart PDHP for $\mathrm{MgCl}_{2}(2.5 \mathrm{mM})$ and $\mathrm{MnCl}_{2}(1.8 \mathrm{mM})$ are similar (Wieland, 1983), but in the case of bovine adrenal PDHP the constants $(1.20$ and $0.35 \mathrm{mM}$, respectively) differ over 3-fold, and a higher PDHP 
activity was observed in the presence of $\mathrm{Mn}^{2+}$ compared to $\mathrm{Mg}^{2+}$ (Strumiło, 1983). In addition to $\mathrm{Mg}^{2+}$, the isoform $\mathrm{PDHP}_{1}$ requires $\mathrm{Ca}^{2+}$ at micromolar concentrations. $\mathrm{Ca}^{2+}$, besides its participation in the interaction of $\mathrm{PDHP}_{1}$ with the $\mathrm{LD}_{2}$ domain, strongly stimulates $\mathrm{PDHP}_{1}$ activity (Huang et al., 1998; Roche et al., 2003). Moreover, a specific regulatory protein for PDHP ${ }_{1}$ has been isolated and partially characterized (Lawson et al., 1997). According to the recently obtained data, $\mathrm{PDHP}_{1}$ is more sensitive to $\mathrm{Mg}^{2+}$ than is $\mathrm{PDHP}_{2}$ (Harris et al., 2002). Polyamines, especially spermine, markedly decrease the $K_{\mathrm{m}}$ values for $\mathrm{Mg}^{2+}$ of both PDHP isoenzymes (Roche et al., 2003). In general, the short-term regulation of PDHP activity is not so varied as is the regulation of PDHK. PDHP activity is influenced mainly by divalent cations. For example, muscle contraction causes an increase in mitochondrial $\mathrm{Ca}^{2+}$ which can then stimulate $\mathrm{PDHP}_{1}$ activity and the whole PDHC (Harris et al., 2002; Patel \& Korotchkina, 2003). Insulin can stimulate PDHPs via their phosphorylation by protein kinase C8, which is activated and translocated to mitochondria of muscle and liver cells by the hormone (Caruso et al., 2001). In additional to the short-term regulation by divalent ions, polyamines and, probably, insulin, a long-term regulation of PDHP via changes in its expression at the genetic level is a confirmed fact. Starvation and diabetes decrease $\mathrm{PDHP}_{1}$ expression, especially in rat heart and kidney (Huang et al., 2003), whereas insulin increases it (Harris et al., 2002; Holness \& Sugden, 2003).

\section{ISOSTERIC INHIBITION BY END-PRODUCTS}

In numerous kinetic studies of PDHC isolated from varied mammalian tissues, its competitive inhibition by acetyl-CoA and NADH versus CoA and NADH has been shown (Wieland, 1983; Yeaman, 1989; Czygier \& Strumiło, 1994). The $K_{\mathrm{i}}$ values for $\mathrm{NADH}$ were in the same range as the $K_{\mathrm{m}}$ values for $\mathrm{NAD}^{+}$. It has been suggested that $\mathrm{NADH}$ inhibition of PDHC at the isosteric level (at the active sites of the E3 component) is important for the control of mammalian PDHC activity (Wieland, 1983). The inhibition of E2 caused by acetyl-CoA may be a little less significant for PDHC regulation since the $K_{i}$ values for acetyl-CoA were usually higher than the $K_{\mathrm{m}}$ values for CoA (Wieland, 1983). For example, in the case of PDHC from the human heart the difference was nearly 3-fold (Kiselevsky et al., 1990). Yet in such states as starvation or diabetes when the mitochondrial ratios of acetyl-CoA/CoA and NADH/ $\mathrm{NAD}^{+}$are very high in some tissues, acetylation and reduction of lipoyl groups in backward reactions catalyzed by E2 and E3 can lead to an inhibition of pyruvate decarboxylation catalyzed by E1 (Wieland, 1983; Patel \& Korotchkina, 2003). The end-prod- uct inhibition at the active centers of the E2 and E3 components seems to be an essential addition to the allosteric regulation of $\mathrm{PDH}$ kinase by acetyl-CoA and NADH, as discussed above. Both mechanisms concern the same, universal and clear principle of regulation - "negative feedback inhibition".

\section{ALLOSTERIC REGULATION BY SOME COFACTORS}

Many investigators have considered that TDP plays only a catalytic role in the active sites of E1. For a long time it has been suggested that mammalian PDHC loses TDP almost completely during isolation, because the measured PDHC activity was usually very low in the absence of added TDP (Walsh et al., 1976; Sumegi \& Alkonyi, 1983). The measurements were based on the initial rate of the reaction catalyzed by PDHC. However, a far higher PDC activity was observed when the reaction rate was measured for a longer period, because in the absence of added TDP the maximum rate was achieved only after a lag-phase of several minutes (Strumiło, 1988). It was established that the duration of the lag-phase and subsequent reaction rate depends strongly on the concentration and type of divalent ions present $\left(\mathrm{Mg}^{2+}, \mathrm{Ca}^{2+}, \mathrm{Mn}^{2+}\right)$. The shortest lag-phase and the highest activity of PDHC with endogenous TDP is observed in the presence of $\mathrm{Mn}^{2+}$ (Czerniecki \& Czygier, 2001). Kinetics studies of bovine, bison, and pig heart PDHC saturated with endogenous TDP in the active sites of E1 showed that the addition of exogenous TDP leads to a disappearance of the lag-phase and manifold reduction of the $K_{\mathrm{m}}$ value for pyruvate, but a more moderate decrease of the $K_{\mathrm{m}}$ for $\mathrm{CoA}$ and $\mathrm{NAD}^{+}$with no considerable change in the maximum reaction rate (Strumiło et al., 1996; 1999; Strumiło \& Czygier, 1998). The apparent $K_{\mathrm{m}}$ value for TDP is about $0.2 \mu \mathrm{M}$ which means that the cofactor is very effective. Generally, an increase of an enzyme's affinity for substrates without alteration in the $V_{\max }$ are typical signs of positive allosteric modulators in view of the model of Monod et al. (1965). TDP acts exactly in such a manner. For example, the $K_{\mathrm{m}}$ values of pig heart PDHC for pyruvate in the absence and presence of added TDP are $76.7 \pm 6.6$ and $19.0 \pm 2.7 \mu \mathrm{M}$, respectively (Strumiło et al., 1999). The increase of PDHC affinity for the substrate is 4fold. It has also been established that TDP added to PDHC changes both the UV and circular dichroism spectra, and lowers the fluorescence emission of the complex containing bound molecules of endogenous TDP in its active centers (Strumiło et al., 2002). The spectral data indicate conformational changes of the PDHC. Thus, TDP as an allosteric modulator can quickly induce conformational and functional changes of the multienzyme complex. The divalent ions 


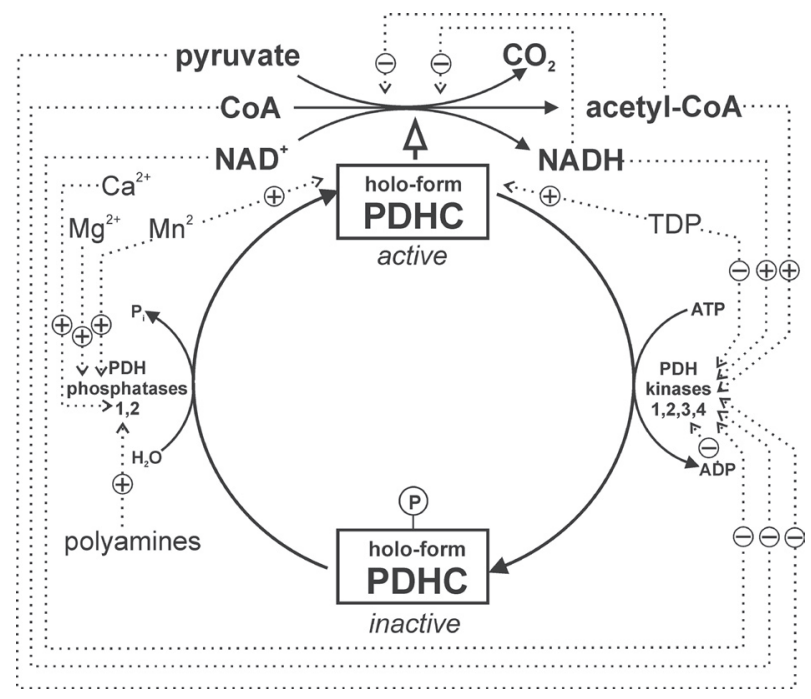

Figure 2. Short-term regulation of the mammalian pyruvate dehydrogenase complex by main effectors.

$\mathrm{Mg}^{2+}, \mathrm{Ca}^{2+}$, and especially $\mathrm{Mn}^{2+}$ increase the effect of TDP (Czerniecki \& Czygier, 2001). Participation of the main effectors in the control of PDHC activity is summarized in Fig. 2.

The data obtained suggest that TDP plays, in addition to its catalytic function, the important role of a positive regulatory effector of mammalian PDHC. Researchers who have the possibility of structural investigations by modern methods ought to notice the allosteric regulation of PDHC. Until now, the structural studies of pyruvate dehydrogenase have been limited to the processes in the active sites of the enzyme and the interactions among its subunits (Ciszak et al., 2003; Korotchkina et al., 2004).

\section{Acknowledgements}

I thank Mr. Jan Czerniecki for his help in computer construction of the figures.

\section{REFERENCES}

Bao H, Kasten SA, Yan X, Hiromasa Y, Roche TE (2004a) Pyruvate dehydrogenase kinase isoform 2 activity stimulated by speeding up the rate of dissociation of ADP. Biochemistry 43: 13442-13451.

Bao H, Kasten SA, Yan X, Roche TE (2004b) Pyruvate dehydrogenase kinase isoform 2 limited and further inhibited by slowing down the rate of dissociation of ADP. Biochemistry 43: 13432-13441.

Bowker-Kinley MM, Davis WI, Wu P, Harris RA, Popov KM (1998) Evidence for existence of tissue-specific regulation of the mammalian pyruvate dehydrogenase complex. Biochem J 329: 191-196.

Caruso M, Maitan MA, Bifulko G, Miele C, Vigliotta G, Oriente F, Formisano P, Beguinot F (2001) Activation and mitochondrial translocation of protein kinase Cठ are necessary for insulin stimulation of pyruvate dehy- drogenase complex activity in muscle and liver cells. $I$ Biol Chem 276: 45088-45097.

Chen G, Wang L, Liu S, Chang C, Roche TE (1996) Activated function of the pyruvate dehydrogenase phosphatase through $\mathrm{Ca}^{2+}$-facilitated binding to the inner lipoyl domain of the dihydrolipoyl acetyltransferase. J Biol Chem 271: 28064-28070.

Ciszak EM, Korotchkina LG, Dominiak PM, Sidhu S, Patel MS (2003) Structural basis for flip-flop action of thiamin pyrophosphate-dependent enzymes revealed by human pyruvate dehydrogenase. J Biol Chem 278: 21240-21246.

Czerniecki J, Czygier M (2001) Cooperation of divalent ions and thiamine diphosphate in regulation of the function of pig heart pyruvate dehydrogenase complex. J Nutr Sci Vitaminol (Tokyo) 47: 385-386.

Czygier M, Strumiło S (1994) Basic properties of the pyruvate dehydrogenase complex isolated from aurochs heart. Acta Biochim Polon 41: 453-457.

Czygier M, Strumiło S (1995a) An investigation of the kinase activity of aurochs heart pyruvate dehydrogenase complex. Biochem Mol Biol Int 37: 313-317.

Czygier MB, Strumiło SA (1995b) Changes in some properties of the aurochs heart pyruvate dehydrogenase complex induced by its partial phosphorylation (in English). Ukr Biokhim Zh 67: 25-28.

Denton RM, McCormack JG, Rutter GA, Burnett P, Edgell NJ, Moule SK, Diggle TA (1996) The hormonal regulation of pyruvate dehydrogenase complex. Adv Enzyme Regul 36: 183- 198.

Fries M, Jung HI, Perham RN (2003) Reaction mechanism of the heterotetrameric (alpha2beta2) E1 component of 2-oxo acid dehydrogenase multienzyme complexes. Biochemistry 42: 6996-7002.

Gudi R, Bowker-Kinley MM, Kedishvili NY, Zhao Y, Popov KM (1995) Diversity of the pyruvate dehydrogenase kinase gene family in humans. J Biol Chem 270: 2898928994.

Harris RA, Huang B, Wu P (2001) Control of pyruvate dehydrogenase kinase gene expression. Adv Enzyme Regul 41: 269-288.

Harris RA, Bowker-Kinley MM, Huang B, Wu P (2002) Regulation of the activity of the pyruvate dehydrogenase complex. Adv Enzyme Regul 42: 249-259.

Hiromasa Y, Fujisawa T, Aso Y, Roche TE (2004) Organization of the cores of the mammalian pyruvate dehydrogenase complex formed by E2 and E2 plus E3-binding protein and their capacities to bind the E1 and E3 components. I Biol Chem 279: 6921-6933.

Holness MJ, Sugden MC (2003) Regulation of pyruvate dehydrogenase complex by reversible phosphorylation. Biochem Soc Trans 31: 1143-1151.

Huang B, Gudi R, Wu P, Harris RA, Hamilton J, Popov KM (1998) Isoenzymes of pyruvate dehydrogenase phosphatase. DNA-derived amino acid sequences, expression, and regulation. J Biol Chem 273: 17680-17688.

Huang B, Wu P, Popov KM, Harris RA (2003) Starvation and diabetes reduce the amount of pyruvate dehydrogenase phosphatase in rat heart and kidney. Diabetes 52: 1371-1376.

Karpova T, Danchuk S, Kolobova E, Popov KM (2003) Characterization of the isozymes of pyruvate dehydrogenase phosphatase: implications for the regulation of pyruvate dehydrogenase activity. Biochim Biophys Acta 1652: 126-135.

Khailova LS, Korochkina LG, Severin SE (1990) Intersite cooperativity in enzyme action of pyruvate dehydrogenase. In Biochemistry and Physiology of TDP Enzymes. Bisswanger H, Ullrich J, eds, pp 251-261, VCH Weinheim, Blaubeuren, Germany. 
Kiselevsky YV, Ostrovtsova SA, Strumiło SA (1990) Kinetic characterization of the pyruvate and oxoglutarate dehydrogenase complexes from human heart. Acta Biochim Polon 37: 135-139.

Kolobova E, Tuganova A, Boulatnikov I, Popov KM (2001) Regulation of pyruvate dehydrogenase activity through phosphorylation at multiple sites. Biochem J 358: 69-77.

Korotchkina LG, Patel MS (2001) Probing the mechanism of inactivation of human pyruvate dehydrogenase by phosphorylation of three sites. J Biol Chem 276: 57315738.

Korotchkina LG, Ciszak EM, Patel MS (2004) Function of several critical amino acids in human pyruvate dehydrogenase revealed by its structure. Arch Biochem Biophys 429: 171-179.

Lawson JE, Park SH, Mattison AR, Van J, Reed LJ (1997) Cloning, expression, and properties of the regulatory subunit of bovine pyruvate dehydrogenase phosphatase. I Biol Chem 272: 31625-31629.

Linn TC, Pettit FH, Reed LJ (1969) $\alpha$-Keto acid dehydrogenase complexes. X. Regulation of the activity of the pyruvate dehydrogenase complex from beef kidney mitochondria by phosphorylation and dephosphorylation. Proc Natl Acad Sci USA 62: 234-241.

Liu S, Baker JC, Roche TE (1995) Binding of the pyruvate dehydrogenase kinase to recombinant constructs containing the inner lipoyl domain of the dihydrolipoyl acetyltransferase component. J Biol Chem 270: 793-800.

Monod J, Wyman J, Changeux J-P (1965) On the nature of allosteric transitions: a plausible model. J Mol Biol 12: 88-117.

Patel MS, Korotchkina LG (2003) The biochemistry of the pyruvate dehydrogenase complex. Biochem Mol Biol Education 31: 5-15.

Perham RN (2000) Swinging arms and swinging domains in multifunctional enzymes: catalytic machines for multistep reactions. Annu Rev Biochem 69: 961-1004.

Reed LJ (2001) A trail of research from lipoic acid to $\alpha$ keto acid dehydrogenase complexes. J Biol Chem 276: 38329-38336.

Roche TE, Hiromasa Y, Turkan A, Gong X, Peng T, Yan X, Kasten SA, Bao H, Dong J (2003) Essential roles of lipoyl domains in the activated function and control of pyruvate dehydrogenase kinases and phosphatase isoform 1. Eur I Biochem 270: 1050-1056.

Steussy CN, Popov KM, Bowker-Kinley MM, Sloan RB Jr, Harris RA, Hamilton JA (2001) Structure of pyruvate dehydrogenase kinase. Novel folding pattern for a serine protein kinase. J Biol Chem 276: 37443-37450.

Strumiło SA (1983) Study of the reactivation of a partially phosphorylated pyruvate dehydrogenase complex from bovine adrenal glands. Biokhimiya 48: 1879-1883 (English edition as Biochemistry - Moscow 48: 1617-1620).

Strumiło SA (1988) Hysteresis behaviour of the pyruvate dehydrogenase complex containing endogenous thiamin pyrophosphate. In Thiamin Pyrophosphate Biochemistry. Schellenberger A, Schowen RL eds, pp 83-91. CRC Press, Boca Raton, Florida.

Strumiło S, Czygier M (1998) Regulation of bovine pyruvate dehydrogenase complex by thiamine pyrophosphate. Biochem Arch 14: 27-32.

Strumiło SA, Senkevich SB, Vinogradov VV (1981) Characterization of kinase activity of pyruvate dehydrogenase complex from bovine adrenals. Biokhimiya 46: 974-978 (English edition as Biochemistry - Moscow 46: 788-792).

Strumiło SA, Senkevich SB, Selevich MI, Vinogradov VV (1984) Inhibition of the kinase activity of the pyruvate dehydrogenase complex of the adrenals by the mitochondrial component. Biokhimiya 49: 155-159 (English edition as Biochemistry - Moscow 49: 132-136).

Strumiło S, Czerniecki J, Dobrzyń P (1999) Regulatory effect of thiamin pyrophosphate on pig heart pyruvate dehydrogenase complex. Biochem Biophys Res Commun 256: 341-345.

Strumiło S, Czygier M, Kondracikowska J, Dobrzyń P, Czerniecki J (2002) Kinetic and spectral investigation of allosteric interaction of coenzymes with 2-oxo acid dehydrogenase complexes. J Mol Struct 614: 221-226.

Sumegi B, Alkonyi I (1983) Elementary steps in the reaction of the pyruvate dehydrogenase complex from pig heart. Kinetic of thiamine diphosphate binding to the complex. Eur J Biochem 136: 347-352.

Walsh DA, Cooper RH, Denton RM, Bridges BJ, Randle PJ (1976) The elementary reactions of the pig heart pyruvate dehydrogenase complex. A study of the inhibition by phosphorylation. Biochem J 157: 41-67.

Wałajtys EI, Gottesman DP, Williamson JR (1974) Regulation of pyruvate dehydrogenase in rat liver mitochondria by phosphorylation-dephosphorylation. I Biol Chem 249: 1857-1865.

Wieland OH (1983) The mammalian pyruvate dehydrogenase complex: structure and regulation. Rev Physiol Biochem Pharmacol 96: 123-170.

Wieland OH, Jagow-Westermann B (1969) ATP-dependent inactivation of heart muscle pyruvate dehydrogenase and reactivation by $\mathrm{Mg}^{++}$. FEBS Lett 3: 271-274.

Wu P, Blair PV, Sato J, Jaskiewicz J, Popov KM, Harris RA (2000) Starvation increases the amount of pyruvate dehydrogenase kinase in several mammalian tissues. Arch Biochem Biophys 381: 1-7.

Yeaman SJ (1989) The 2-oxo acid dehydrogenase complexes: recent advances. Biochem I 257: 625- 632 